\title{
Kleinkapazitäre Blase: Medikamente und/oder Miktionstraining?
}

\author{
Low Capacity Bladder: Dugs and/or Toilet Training
}

Autoren

Institute

\section{A. Wiedemann ${ }^{1,2}$, H. J. Heppner ${ }^{2,3}$}

${ }^{1}$ Urologische Abteilung, Evangelisches Krankenhaus Witten

${ }^{2}$ Lehrstuhl für Geriatrie der Universität Witten/Herdecke

${ }^{3}$ Klinik für Geriatrie, Helios-Klinikum Schwelm

\author{
Schlüsselwörter \\ - Harninkontinenz \\ - Überaktive Blase \\ - Miktionstraining \\ - medikamentöse Therapie \\ - Verhaltenstherapie \\ Key words \\ - urinary incontinence \\ - overactive bladder \\ - toilet training \\ - drug therapy \\ - behavioral intervention
}

\section{Bibliografie}

DOI http://dx.doi.org/ 10.1055/s-0035-1550037

Online-Publikation: 4.8.2015

Akt Urol 2015; 46: 378-381

(c) Georg Thieme Verlag KG

Stuttgart · New York

ISSN 0001-7868

\section{Korrespondenzadresse}

Priv.-Doz. Dr. med. Andreas

Wiedemann

Urologische Abteilung

Evangelisches Krankenhaus im

Diakoniewerk Ruhr gGmbH

Lehrstuhl für Geriatrie der

Universität Witten

Pferdebachstraße 27-43

58455 Witten

Tel.: + 49/2302/175 2521

Fax: +49/2302/175 2075

awiedemann@diakonie-ruhr.de

\section{Zusammenfassung}

\section{$\nabla$}

Hintergrund: Verhaltenstherapeutische Maßnahmen werden im Zusammenhang mit der Standardtherapie einer Überaktiven Blase genannt; letztendlich ist ihr Stellenwert in der Therapie jedoch unklar.

Methoden: Systematische Literaturrecherche zum Thema unter den Stichworten „overactive bladder“ and „behavioral therapy“. Berücksichtigung fanden Originalarbeiten mit Untersuchungen an Erwachsenen mit englischem Abstract. Ergebnisse: Im Einzelnen werden die Ergebnisse von 6 Studien vorgestellt, die eine Verhaltensintervention als singuläre Therapieform bei einer Überaktiven Blase einsetzen, einen Vergleich mit einer medikamentösen Standardtherapie mit einem Antimuskarinikum anstellen bzw. die Verhaltensintervention mit einem Miktionstraining als Augmentation bei einem ungenügenden Ansprechen der Symptomatik auf eine anticholinerge Therapie erlauben. Die Eingangskriterien mit der Definition der Überaktiven Blase, Patientenklientel und Studiendesign bzw. -dauer und die Art der Verhaltensintervention bzw. die hierzu verwendeten Medien sind wie die gefundenen Ergebnisse uneinheitlich. So wird die Verhaltensintervention als alleinige Maßnahme als wirksam erachtet; manche Autoren sehen einen positiven Effekt der zusätzlich zur anticholinergen Therapie angebotenen Verhaltensintervention, andere Autoren sehen einen solchen Effekt nicht.

Schlussfolgerung: Obwohl experimentelle Untersuchungen über die neuronalen Mechanismen der cerebralen Verarbeitung des Harndranges und der Auslösung des Miktionsreflexes die potentielle Wirksamkeit von verhaltenstherapeutischen Maßnahmen als wahrscheinlich annehmen lassen, ist das Bild der hierzu gefundenen Literatur uneinheitlich.

\section{Abstract \\ $\nabla$}

Background: Behavioral interventions are part of the standard therapy for the overactive bladder syndrome although its impact still is unclear. Methods: A systematic review according to the key words „overactive bladder“ and "behavioral therapy” was undertaken. Original studies dealing with adults and with an English abstract were taken into consideration.

Results: Due to these requirements, only 6 studies were found. They use behavioral interventions as single therapy or compare a behavioral intervention with a standard drug therapy. On the one hand this was a direct comparison to a therapy with antimuscarinics, on the other hand drug therapy was augmented by behavioral interventions if the primary therapy failed. Inclusion criteria, patient characteristics, methods of behavioral intervention, the kind of media used and the results were inconsistent. As a result behavioral interventions are considered to be effective in controlling symptoms of an overactive bladder; in some studies an additive effect to drug therapy is seen if behavioral interventions are performed as supplementary measure; other studies could not confirm such an effect. Conclusion: Although experimental studies about micturition control in the CNS make a positive effect of behavioral interventions in overactive bladder more than likely, the literature reports on this topic remain inhomogeneous. 


\section{Einleitung}

Die Therapie der Überaktiven Blase besteht in erster Linie aus konservativen und medikamentösen Therapieansätzen $[1,2]$. Operative Maßnahmen wie die sakrale Neuromodulation oder der Harnblasenersatz bleiben seltenen Extremvarianten vorbehalten. Zu den konservativen Maßnahmen gehören die Analyse und Beeinflussung des Trinkverhaltens laut einem Trink- und Miktionsprotokoll, die Behandlung einer eventuellen Obstipation und das Vermeiden von Reizstoffen wie Nikotin, Pfeffer, scharfe Gewürze oder säurehaltige Getränke aber auch ein unterstützendes „Miktionstraining“ [3]. Gemeint ist neben „festen Entleerungszeiten“, „,individuellen Entleerungszeiten“ und dem „angebotenen Toilettengang“ vor allem das „Blasentraining“, bei dem das selbstständige Gehen zur Toilette nach einem individuell festgelegten und veränderten Plan in bestimmte verhaltenstherapeutische Schulungsmaßnahmen eingebettet wird ( $\bullet$ Tab. 1). Unter den genannten „Rehabilitations- und Schulungstechniken“ sind Informationen über die physiologische Häufigkeit und Menge der Miktion, der Trinkmenge und Blasenfunktion im Allgemeinen gemeint. Weiterhin ist Bestandteil eines solchen Programmes das Führen eines Miktionstagebuches und damit die Bewusstmachung der Miktionsfrequenz und Harndrangvermeidungsstrategien. Hierunter wird die „Verarbeitung“ des Harndranges mit einem Wegführen der Gedanken vom Harndrang etwa durch einen „Themenwechsel“, Zählen oder Singen verstanden.

Rationale dieses Vorgehens ist das Begreifen der Miktion auch als „koordinierten Reflex“. Im physiologischen Erregungsablauf, der zu einer Miktion führt, erreichen afferente Impulse über den Füllungszustand der Blase das periaquäduktale Grau der Pons. Dieses pontine Miktionszentrum verarbeitet die ankommenden Impulse, leitet die Miktion durch Aktivierung von Motoneuronen der Blase und Hemmung des Sphinkter externus ein, sorgt aber auch für eine Sphinkteraktivierung in der Speicherphase der Blase [5-7]. MRT-Untersuchungen an Frauen, die ein verändertes cerebrales Reaktionsmuster nach Beckenbodentraining während einer Provokationsfüllung der Harnblase demonstrieren, lassen annehmen, dass diese cerebralen Abläufe des Miktionsreflexes modulierbar sind $[8,9]$.

\section{Methoden}

$\nabla$

Zur Bestimmung der Wertigkeit eines Miktionstrainings und der Frage, ob ergänzend zur klassischen medikamentösen Therapie einer Überaktiven Blase ein Miktionstraining eingesetzt werden soll bzw. ob sich ein Versuch vor dem Einsatz von Antimuskarinika lohnt oder eher eine Augmentation einer medikamentösen Therapie durch ein Miktionstraining bei Nichtansprechen oder ungenügender Symptomkontrolle sinnvoll ist, wurde eine Literaturrecherche in medline unter den Stichworten „overactive bladder“ und „behavioral training“ vorgenommen. Von den 83 Treffern fanden im Folgenden nur Originialarbeiten mit engl. Abstract, die sich mit einem Miktionstraining bei Erwachsenen beschäftigen, Berücksichtigung. Sie werden im Folgenden besprochen.

\section{Ergebnisse}

In einer prospektiven Untersuchung an 108 Patienten mit idiopathischer Überaktiver Blase, von denen 85 die Testphase beendeten (38 Männer, 47 Frauen), untersuchte Lee die Effekte von 2 jeweils 30 minütigen Schulungen über die physiologischen Miktionsabläufe, Anzahl und Volumen der normalen Miktionen. Es wurde ein Video über die Miktions- und Speicherphase der Harnblase gezeigt und das Ziel verdeutlicht, die Miktionsabstände langsam zu verlängern. Genannte Strategien waren das Vermeiden des sofortigen Aufsuchens der Toilette und des Denkens an den Harndrang durch Wechseln des Gedankeninhaltes gefolgt von Beckenbodenkontraktionen. Die Schulungen enthielten ein individuelles Gespräch mit einer geschulten Pflegekraft. Eine bestehende medikamentöse Therapie wurde nicht verändert. Nach der 2. Schulung beurteilten 48,7\% der Patienten die Therapie als effektiv und weitere $46,2 \%$ als sehr effektiv. 30,8\% der Patienten gaben eine 50-75\%ige Symptomreduktion und weitere 20,5 eine 75-100\%ig Symptomkontrolle an. 25,5\% der Patienten fanden, dass das Vermeiden des Ganges zur Toilette, 20,5\% das Vermeiden des Denkens an Harndrang und 38,5\% das Kontrahieren des Beckenbodens am meisten hilfreich war. Die Punktwerte im OAB-q (OAB-Questionnaire), IPSS (Internationaler ProstataSymptomenscore), ICI-q (Questionnaire der International Consultion on Inctontinence) zeigten eine jeweils signifikante Reduktion zwischen den Ausgangswerten und der ersten Visite nach der ersten Schulung [10]. Die Autoren schlussfolgerten, dass ein Miktionstraining in der Lage ist, Symptome einer Überaktiven Blase $z u$ verbessern.

Wyman standardisierte bei 357 Patienten die medikamentöse Therapie, indem er das bisher gegebene Antimuskarinikum auf Tolterodin $4 \mathrm{mg}$ vereinheitlichte und ergänzend ein Miktionstraining durchführte. Eingeschlossen wurden Patienten über 18 Jahre mit Symptomen einer Überaktiven Blase über mehr als 3 Wochen definiert als $\geq 8$ Miktionen am Tag und $\geq 2$ Drangepiso-

Tab. 1 Verschiedene Formen des Toilettentrainings in Abhängigkeit von kognitiven und funktionellen Ressourcen des Patienten, Evidenzlevel und Empfehlungsklassen It. Leitlinie Harninkontinenz der Dt. Gesellschaft für Geriatrie [4].

\begin{tabular}{|c|c|c|c|}
\hline Form & Durchführung & Zielgruppe & Evidenzgrad/Empfehlung \\
\hline $\begin{array}{l}\text { Festgelegte Entleerungszeiten } \\
\text { (timed voiding) }\end{array}$ & z. B. alle 2 Stunden Begleitung zur Toilette & $\begin{array}{l}\text { kognitiv Eingeschränkte, funktionell } \\
\text { Abhängige }\end{array}$ & $\mathrm{IV} / \mathrm{C}$ \\
\hline $\begin{array}{l}\text { Individuelle Entleerungszeiten } \\
\text { (habit training) }\end{array}$ & wie oben, jedoch mit individuellem Toilettenplan & $\begin{array}{l}\text { kognitiv Eingeschränkte, funktionell Abhän- } \\
\text { gige, wenn Miktionsmuster festellbar }\end{array}$ & $\mathrm{Ila} / \mathrm{B}$ \\
\hline $\begin{array}{l}\text { Angebotener Toilettengang } \\
\text { (prompted voiding) }\end{array}$ & $\begin{array}{l}\text { Aufmerksamkeit wird auf die Blase gelenkt, } \\
\text { Rückmeldung gegeben, Toilettengang angebo- } \\
\text { ten, verbale positive Rückmeldung, Bemerkung } \\
\text { über Zeitpunkt des nächsten Toilettenganges }\end{array}$ & $\begin{array}{l}\text { Nicht ausreichende kognitive Fähigkeiten } \\
\text { für komplexe Interventionen } \\
\text { Aber Toilette kann benutzt werden, } \\
\text { Harndrang wird gespürt }\end{array}$ & $\mathrm{IIb} / \mathrm{A}$ \\
\hline $\begin{array}{l}\text { Blasentraining (bladder } \\
\text { drill) }\end{array}$ & $\begin{array}{l}\text { selbstständiges Gehen zur Toilette nach } \\
\text { individuellem Plan, aktive Rehabilitations- und } \\
\text { Schulungstechniken }\end{array}$ & $\begin{array}{l}\text { Kognitiv Kompetente, Motivierte, } \\
\text { Eigeninitiative vorhanden }\end{array}$ & $\mathbf{I b} \mid \mathbf{A}$ \\
\hline
\end{tabular}


den in $24 \mathrm{~h}$ und $\geq 1$ Drangkontinenzepisoden in 5 Tagen in Kombination mit einer Unzufriedenheit mit der bisherigen antimuskarinergen Therapie. Alle Patienten wurden einheitlich auf Tolterodin $4 \mathrm{mg}$ täglich umgestellt und erhielten eine schriftliche Anleitung zum Miktionstraining, die mündlich erklärt wurde. Eine Evaluation fand 8 Wochen später statt. Zu diesem Zeitpunkt noch nicht zufriedenstellend therapierte Patienten erhielten ein intensives Gespräch mit einem Arzt zur Verbesserung des Miktionstrainings und wurden nach weiteren 8 Wochen untersucht. Nach 8 bzw. 16 Wochen waren 201 bzw. 252 Patienten (53 bzw. $64 \%$ ) sehr zufrieden mit dem Therapieeffekt. Von den 33 Patienten, die die Therapie nach der 8. Woche mit dem „Standardmiktionstraining “ als nicht zufriedenstellend einstuften, ließen sich $25(76 \%)$ durch das individualisierte Training noch konvertieren. Alle Variablen des Miktionsprotokolles und des OAB-q-Scores verbesserten sich vom Ausgangswert bereits zur 4. Therapiewoche signifikant $(p<0,0001)$ [11]. Die Autoren werteten die Ergebnisse als Beleg für die positiven Effekte eines zusätzlich zur antimuskarinergen Therapie durchgeführten Miktionstrainings.

Nicht als Sequenz, sondern in einem Vergleich zwischen einer Gruppe von OAB-Patienten (definiert als $\geq 8$ Miktionen am Tag, imperativem Harndrang mit oder ohne Dranginkontinenz) mit alleiniger medikamentöser Therapie oder in Kombination mit einem Miktionstraining setzte Matthiasson die Verhaltensintervention ein [12]. Es wurden 501 Patienten (75\% weiblichen Geschlechts) in 2 Arme aufgeteilt: 257 erhielten Tolterodin $2 \times 2 \mathrm{mg}$ alleinig; 244 Patienten in Kombination mit einem Miktionstraining. Eine Evaluation mit Miktionsprotokoll und dem Volumen pro Miktion fand nach der 2., 12. und 24. Therapiewoche statt. Zum letzten Zeitpunkt war die Reduktion der Miktion in $24 \mathrm{~h}$ mit 33 vs. 25\% in der Kombinationsgruppe signifikant größer als in der nur medikamentös behandelten Gruppe $(\mathrm{p}<0,001)$. Auch das mittlere Miktionsvolumen hatte in der Kombinationsgruppe um 31 \% zugenommen, während bei den nur mit Tolterodin behandelten Patienten dieser Zuwachs nur 20\% betrug $(\mathrm{p}<0,001)$. Die Reduktion der Inkontinenzepisoden fiel mit 87 vs. $81 \%$ in beiden Gruppen vergleichbar aus (n. s.). Die Autoren kamen zu dem Schluss, dass die Wirksamkeit einer medikamentösen Therapie der Überaktiven Blase durch ein ergänzendes Miktionstraining verbessert werden kann.

Im „Male Overactive Bladder Treatment in Veterans Trial“ (MOTIVE) führte Burgio einen randomisierten Vergleich zwischen einer alleinig mit Verhaltenstherapie und einer alleinig medikamentös behandelten Gruppe von 143 Männern zwischen 42 und 88 Jahren durch. Eingangskriterium waren mehr als 8 Miktionen mit imperativem Harndrang mit oder ohne Dranginkontinenz; alle Männer erhielten standardisiert eine alpha-Blocker-Therapie und eine individuelle Titration von 5-30 mg Oxybutynin am Tag. Gemessen wurde die Miktionsfrequenz in $24 \mathrm{~h}$, die Nykturie, die Stärke des Harndranges und der AUA-SymptomenScore. Die Miktionsfrequenz nahm um 18,8\% in der Verhaltenstherapie-Gruppe ab, in der Oxybutynin-Gruppe um 16,9\%. Die statistische Analyse demonstrierte mit einem $\mathrm{p}<0,1$ eine Gleichwertigkeit beider Therapiearme. In dem Arm mit Verhaltenstherapie war die Reduktion der Nykturie stärker $(-0,7$ vs. $-0,32$ Episoden pro Nacht, $p=0,05)$, in dem medikamentös behandelten Therapiearm fiel die Reduktion des Urgency-Scores größer aus ( $-0,44$ vs. $-0,12 ; \mathrm{p}<0,02)$. Andere Unterschiede zwischen beiden Gruppen waren nicht signifikant [13]. Im Fazit der Autoren wurde die antimuskarinerge Therapie und die Verhaltenstherapie als gleichwertig in der Behandlung einer OAB bei Männern und Alpha-Blockade befunden.
Von der gleichen Autorin wurde 2008 eine multizentrische, randomisierte Untersuchung zur Thematik vorgelegt; 307 Frauen mit idiopathischer OAB (mit der klinischen Diagnose einer Dranginkontinenz oder Mischinkontinenz mit führender Drangkomponente wurden in 2 Gruppen eingeteilt: 153 Frauen erhielten Tolterodin $4 \mathrm{mg}$; 154 Frauen Tolterodin $4 \mathrm{mg}$ und eine zusätzliche Verhaltenstherapie. Zielvariable war eine Reduktion der Dranginkontinenzepisoden um mehr als 70\% unter gleichzeitiger Beendigung der medikamentösen Therapie ohne einen Wechsel in ein anderes Therapieregime. In beiden Gruppen konnte dieser Zielwert in $41 \%$ erreicht werden; in der kombiniert behandelten Gruppe war der Anteil von Patientinnen mit einer mehr als 70\%igen Reduktion der Dranginkontienzepisoden mit 69 vs. 58\% statistisch signifikant größer. So lautete die Schlussfolgerung, dass eine zusätzliche Verhaltenstherapie zwar nicht die Chance auf ein Absetzen der medikamentösen Therapie erhöht, aber eine stärkere Reduktion der Dranginkontinenzepisoden mit sich bringt, was für die Wahrnehmung der Patientinnen einen positiven Effekt hat [14].

\section{Diskussion \\ $\nabla$}

Mit der Rolle des pontinen Miktionszentrums mit einer Verarbeitung und Weiterleitung der von der Blase einlaufenden Afferenzen mit einem Bewusstwerden des Harndranges im frontalen Cortex und einer bewussten Entscheidung zur Auslösung des Miktionsreflexes lassen sich Elemente der Verhaltensmedizin auf die Pathophysiologie der Überaktiven Blase übertragen. Sie geht von veränderbaren Reizschwellen, erlerntem Verhalten und dann auch wieder verlernbarem Verhalten durch verhaltenstherapeutische Interventionsmöglichkeiten aus. Dieser Ansatz ist in der Rauchentwöhnung [15], der Gewichtsreduktion [16,17] oder der Therapie des Diabetes mellitus [18] oder arteriellen Hypertonus [19] etabliert. MRT-Befunde der Arbeitsgruppe von Bertil Blok mit einer nachweisbaren Veränderung des Aktivitätsmusters verschiedener Hirnregionen auf eine schnelle Blasenfüllung mit und ohne Beckenbodentraining deuten darauf hin, dass sich tatsächlich mit verhaltenstherapeutischen Interventionen in die neuronale Miktionssteuerung eingreifen und damit auch ein pathologischer Erregungsablauf modulieren lässt.

Die in der vorliegenden Literaturrecherche gefundenen Arbeiten zum Thema sind uneinheitlich im Studiendesign; es wird ein Miktionstraining als solche verhaltenstherapeutische Intervention nicht standardisiert entweder als schriftliche Anleitung übermittelt, eventuell zusätzlich mündlich erläutert oder mit Videos untermauert ein- oder mehrschrittig durchgeführt. Es liegen Untersuchungen mit einer alleinig oder zusätzlich medikamentös therapierten Kontrollgruppe vor - hier wird Tolterodin oder Oxybutynin in variabler oder fixer Dosierung herangezogen oder „eine bestehende anticholinerge Medikation“ unbekannter Art oder Dosierung beibehalten. Die Verhaltenstherapie wird primär vor einer klassischen anticholinergen Therapie, als Alternative oder auch nach Therapieversagen der antimuskarinergen Therapie eingesetzt. Erfolgsparameter sind subjektive Angaben wie die „Zufriedenheit“ mit der Therapie aber auch objektive Parameter wie die Miktionsfrequenz oder der Harndrang gemäß Miktionsprotokoll oder entsprechenden validierten Scores. Einheitliche Eingangskriterien existieren nicht; die meisten Untersuchungen lehnen sich hier an die ICS-Definition der Überaktiven Blase von 2003 an [20]; inwieweit hier der 
Nachsatz „in Abwesenheit einer Infektion oder anderen Ätiologie“ abgeklärt wurde, bleibt vielfach offen. Urodynamisch kontrollierte Studien existieren nicht - weder was die Eingangskriterien einer Überaktiven Blase, noch die Erfolgsparameter betrifft. Dennoch überwiegt der Eindruck in randomisierten Untersuchungen, dass sich mit einer Verhaltensintervention Symptome einer Überaktiven Blase positiv beeinflussen lassen. Von Vorteil erscheint der Umstand, dass ein Miktionstraining kostengünstig und nebenwirkungsfrei erscheint und damit kostbare Ressourcen schont. Das Fehlen von urodynamisch kontrollierten Studien erscheint jedoch in einem Punkt bedenklich: die Indikation zu einem Miktionstraining mit Harndrangvermeidungsstrategien und in der Eskalation mit Beckenbodenkontraktionen wird ohne Kenntnis des nur urodynamisch bestimmbaren intravesikalen Druckes gestellt. Liegt eine Überaktive Blase mit instabilen Detrusorkontraktionen in der terminalen oder phasischen Form vor, bleibt unklar, wie sich dieser intravesikale Druck unter der Therapie verhält. So ist u.U. eine Subgruppe von Patienten mit erhöhtem intravesikalem Druck, dessen Senkung zum Schutz der oberen Harnwege unerlässlich ist, möglicherweise im Falle eines Nichtansprechens der Verhaltensintervention länger oder im Falle eines zunehmenden intravesikalen Druckes sogar unter der Therapie stärker gefährdet. Diese aus funktionsurologischer Sicht essentielle Frage sollte in urodynamisch kontrollierten Untersuchungen geklärt werden, ehe eine generelle Empfehlung zu einem Miktionstraining bei jeder Form der Überaktiven Blase alleine definiert über die Symptomatologie - ausgesprochen werden kann.

Interessenkonflikt: Nein

\section{Literatur}

1 Thuroff JW, Abrams $P$, Andersson KE et al. EAU guidelines on urinary incontinence. Eur Urol 2011; 59: 387-400

2 Abrams $P$, Andersson KE, Birder L et al. Fourth International Consultation on Incontinence Recommendations of the International Scientific Committee: Evaluation and treatment of urinary incontinence, pelvic organ prolapse, and fecal incontinence. Neurourol Urodyn 2010; 29: 213-240

3 Dannecker C, Friese $K$, Stief $C$ et al. Urinary incontinence in women: part 1 of a series of articles on incontinence. Dtsch Arztebl Int 2010; 107: $420-426$
4 Becher $K$, Bojack B, Ege S et al. Gesellschaft für Geriatrie - aktualisierte Fassung September 2009. Eur J Ger 2010; 12: (Suppl 1): 1-52

5 Blok BF, Sturms LM, Holstege G. A PET study on cortical and subcortical control of pelvic floor musculature in women. J Comp Neurol 1997; 389: 535-544

6 Blok BF, Holstege G. The central nervous system control of micturition in cats and humans. Behav Brain Res 1998; 92: 119-125

7 Blok BF, Holstege G. The central control of micturition and continence: implications for urology. BJU Int 1999; 83: (Suppl 2): 1-6

8 Griffiths D, Clarkson B, Tadic SD et al. Brain Mechanisms Underlying Urge Incontinence and its Response to Pelvic Floor Muscle Training. J Urol 2015

9 Tadic SD, Griffiths D, Schaefer $W$ et al. Brain activity underlying impaired continence control in older women with overactive bladder. Neurourol Urodyn 2012; 31: 652-658

10 Lee HE, Cho SY, Lee S et al. Short-term Effects of a Systematized Bladder Training Program for Idiopathic Overactive Bladder: A Prospective Study. Int Neurourol J 2013; 17: 11-17

11 Wyman JF, Klutke C, Burgio K et al. Effects of combined behavioral intervention and tolterodine on patient-reported outcomes. Can J Urol 2010; 17: 5283-5290

12 Mattiasson A, Blaakaer J, Hoye K et al. Simplified bladder training augments the effectiveness of tolterodine in patients with an overactive bladder. BJU Int 2003; 91: 54-60

13 Burgio KL, Goode PS, Johnson TM et al. Behavioral versus drug treatment for overactive bladder in men: the Male Overactive Bladder Treatment in Veterans (MOTIVE) Trial. J Am Geriatr Soc 2011; 59: 2209-2216

14 Burgio KL, Goode PS, Richter HE et al. Combined behavioral and individualized drug therapy versus individualized drug therapy alone for urge urinary incontinence in women. J Urol 2010; 184: 598-603

15 Schnoll RA, Goelz PM, Veluz-Wilkins A et al. Long-term Nicotine Replacement Therapy: A Randomized Clinical Trial. JAMA Intern Med 2015

16 Forman EM, Butryn ML. A new look at the science of weight control: how acceptance and commitment strategies can address the challenge of self-regulation. Appetite 2015; 84: 171-180

17 Malone M, Alger-Mayer SA, Anderson DA. The lifestyle challenge program: a multidisciplinary approach to weight management. Ann Pharmacother 2005; 39: 2015-2020

18 Huang CY, Lai HL, Lu YC et al. Risk Factors and Coping Style Affect Health Outcomes in Adults With Type 2 Diabetes. Biol Res Nurs 2015

19 Friedberg JP, Rodriguez MA, Watsula ME et al. Effectiveness of a tailored behavioral intervention to improve hypertension control: primary outcomes of a randomized controlled trial. Hypertension 2015; 65: 440-446

20 Abrams $P$. Describing bladder storage function: overactive bladder syndrome and detrusor overactivity. Urology 2003; 62: (Suppl 2): 28-37 